\title{
Performance estimation of kayak paddlers based on fluctuation analysis of movement signals
}

\author{
Gergely Vadai, Zoltán Gingl, Róbert Mingesz and Gergely Makan \\ Department of Technical Informatics \\ University of Szeged \\ Szeged, Hungary \\ E-mail: vadaig@inf.u-szeged.hu
}

\begin{abstract}
Modern coaching of professional kayak paddlers is supported by on-water measurement devices and scientific analysis techniques. However, it is very difficult to determine perfect paddling technique and the interpretation of the measured signals is rather complex. Therefore it is quite challenging to provide easily usable but still accurate indicators of paddlers' performance. In this paper, we show that fluctuation analysis of movement signals provides that can be used to classify the performance of athletes. The standard deviation and Poincaré plots of classical parameters turned out to be useful new indicators of paddling skills, furthermore the signal to noise ratio calculated from power density spectrum of raw movement signals shows relationship with the quality of paddling technique, too. The detailed analysis have been made for paddlings of 26 athletes with different ages and technical skills, which measured by a universal measurement system developed for this task.
\end{abstract}

Keywords-performance estimation; measurement system; fluctuation analysis; kayaks motion; coaching

\section{INTRODUCTION}

Nowadays it is more and more common to use scientific methods in the preparation of professional athletes, such as developing measurement systems to help coaching, as well in kayak-canoe sport. However, to extract quantitative information about quality of paddling technique or performance is rather difficult, because it is not possible to determine the perfect technique by signal analysis, tenths of seconds decide in a race between athletes using very different paddling techniques. Furthermore, the accuracy of measurement is limited by using inertial sensors for measuring the kayaks motion and additional circumstances like weather or mental condition.

Nevertheless, easily usable indicators could be useful for trainers at the examination of the athletes' technical improvement. Common parameters could be detected from the kayaks motion or further quantities could be measured like heart rate or force in the paddle [1-5]. Unfortunately, estimating the efficiency of performance from the trends of several parameters or from the shape of raw measured data is a quite complex task, and in most cases detailed analysis is too difficult and time consuming for coaches.

In the following, we show that fluctuation of classical

This work was supported by DEAK Plc., EDF DEMÁSZ Zrt. and TÁMOP-4.2.2.A-11/1/KONV-2012-0073 parameters provides further easily usable indicators of technical skills. The fluctuation analysis have been made using 6 degrees of freedom inertial signals measured by an expandable on-water measurement system have developed in our laboratory [6]. Furthermore, we shall discuss the possibility of extracting further indicators from the raw movement signals with spectral analysis.

\section{MEASUREMENT SETUP}

Although there are several devices on the market developed for kayak motion analysis, they have many limitations and raw data is rarely available. We have developed a universal measurement system that allows connection of many different sensors for measuring further relevant quantities. Our off-line analysis software provides the required information to aid the collaboration with coaches and athletes in order to carry out the extensive research to find the best indicators.

The measurement system is based on a mixed-signal microcontroller, which includes a 12 bit analogue-to-digital converter. The device measures the kayaks' motion with a built-in 3-axis gyroscope and 3-axis accelerometer, and the data are saved to a $4 \mathrm{~GB}$ micro SD card, which allows long active work for several hours. The device operates from a rechargeable battery. The measured data can be uploaded to the PC via USB 2.0 dongle that provides charging of the battery as well. The analysis of the raw motion signals is done off-line on the PC by our JAVA based software. This application allows

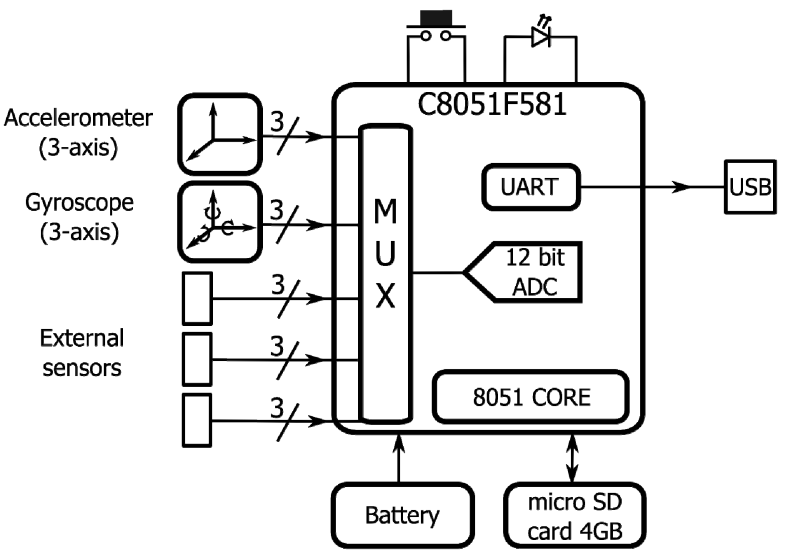

Fig. 1. Block diagram of the developed device 
setting parameters like sensor configuration, sample rate, which was $1000 \mathrm{~Hz}$ in our measurements. The block diagram of the system is shown on Fig. 1. Detailed information of the device is presented in another publication [6].

In the present analysis, we have examined the paddling of 26 athletes with different age and technical skills. The paddlings have been found very different: at training the purpose is producing steady performance, while at race the task is paddling as fast as possible. In order to compare the athletes, 80 seconds long paddling (produced at training) periods have been studied, the transients were skipped.

Accurate reconstruction of the motion using the measured inertial signals is not possible because of the limited precision of the measurement. However one can get information about the performance by examining the shape of the signals and by using statistical analysis. The classical parameters of a stroke cycle can be calculated from the x-axis (forward) acceleration signal using peak search and level crossing algorithms. Note
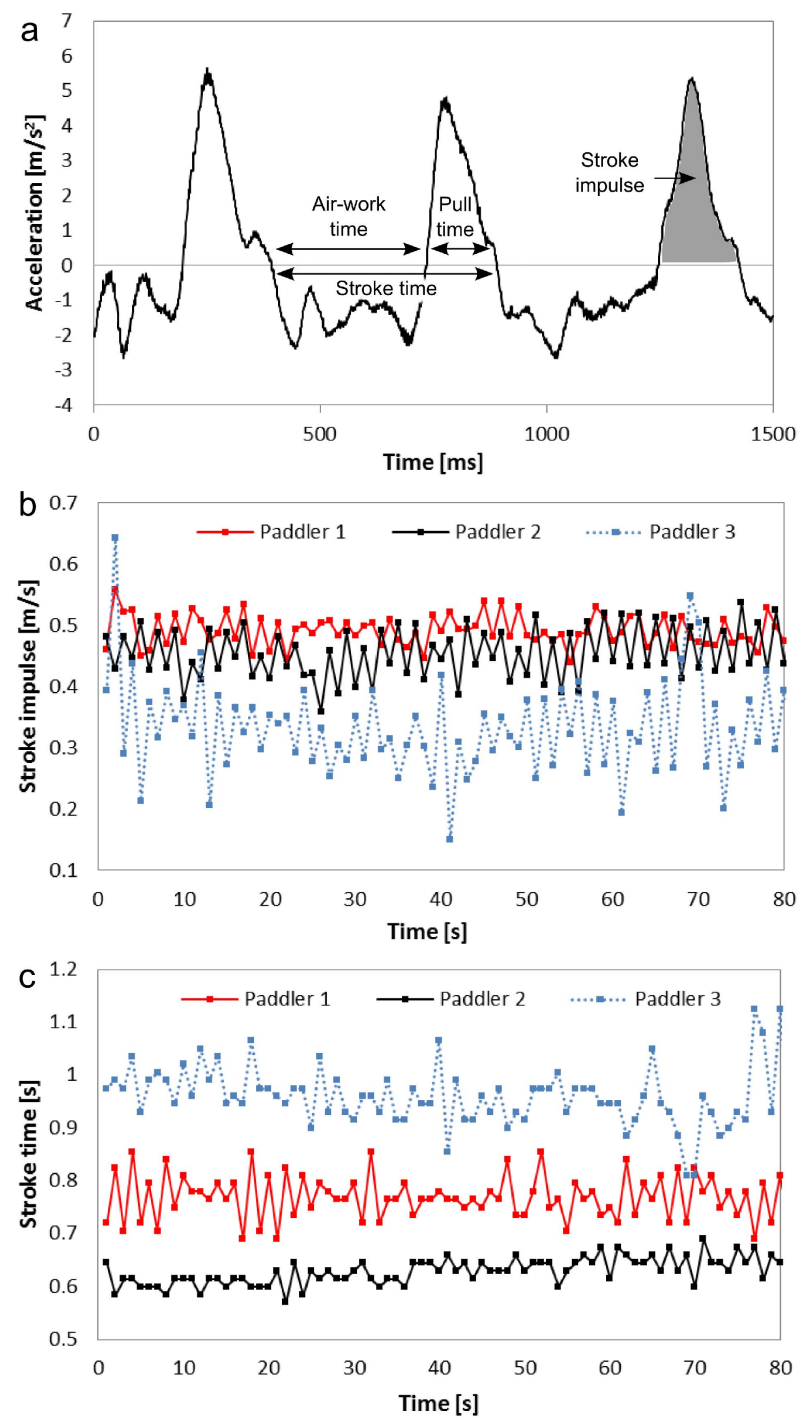

Fig. 2. (a) Parameters of a stroke cycle detected in $\mathrm{x}$-axis acceleration signal. (b) Trend curves of stroke impulse calculated for three athletes' 80 seconds long typical paddling at training. (c) Trend curves of stroke time for the mentioned paddlings. that the measured signals of a technical fault or of a sprint race paddling are more complex, therefore detecting the presented factors with sufficient accuracy is really challenging [6].

The time of a stroke cycle (stroke time) can be divided into two parts: pull time, which represents the time of effective phase of a pulling and air-work time, which is the recovery phase between two pullings (see Fig. 2(a)). We can calculate the increase of the speed caused by the actual pulling by integration of the pull part of the $\mathrm{x}$-axis acceleration signal. This factor is the stroke impulse (see Fig. 2), and it is proved to be a useful parameter for further analysis, furthermore it provides useful information for performance estimation and the calculation of real mechanical impulses is not necessary [6]. Alternative parameters could be defined by using these quantities like stroke rate, total impulse/min or a symmetry factor, which characterizes a personal paddling style.

\section{FLUCTUATION ANALYSIS OF CLASSICAL PARAMETERS}

The interpretation of a stroke cycle and the analysis of the relationship between technical quality and the shape of acceleration signal (or paddling force signal) have been discussed in several works [3, 7-9]. Nevertheless, in most cases it would be very difficult for the coaches to analyze the shape of raw signals. In the following we will show that the temporal fluctuation of stroke time and stroke impulse provides easily usable but still accurate indicators of the quality of paddling.

Fig. 2 (b-c) shows the trend curves of the stroke impulses and stroke times calculated for 80 seconds of paddling of three athletes. Paddler 1 and Paddler 2 are professional athletes with symmetric and asymmetric style, respectively. Paddler 3 is a young, less practiced athlete with asymmetric paddling style.
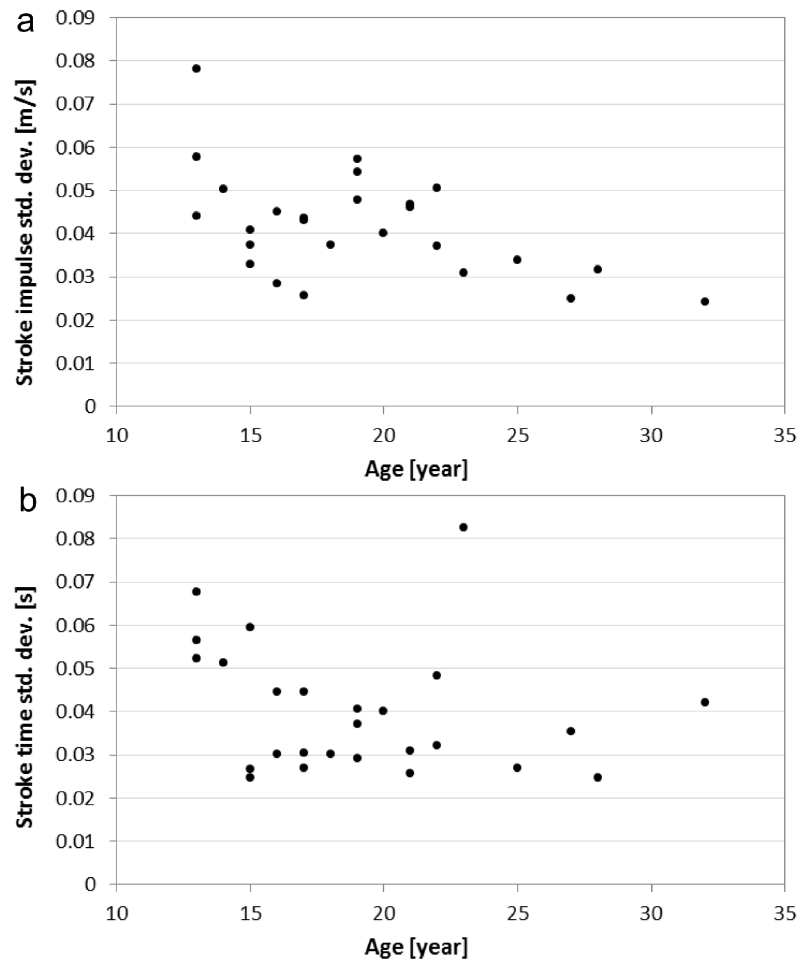

Fig. 3. Standard deviaton of stroke impulse (a) and stroke time (b) as a function of paddlers age. 
As we can see, beyond that the mean of Paddler 3's stroke impulse is not surprisingly smaller than mean impulse of adult paddlers, the parameters of adult paddlers performance is changing slightly, while in the case of Paddler 3 this changing is more intense.

As depicted on Fig. 3, the standard deviation of above mentioned quantities decreases with age significantly, therefore it shows connection between the parameters' fluctuation and technical skills. Important to note that the athletes' age does not characterize the quality of paddling technique perfectly: the technical improving is faster at young ages, but the relationship could be weaker at the case of older athletes. Nevertheless, this is a good demonstration of the evolution of the parameters and provides good indicators of technical skills.

The Poincaré plots of these parameters visualize the examined fluctuation spectacularly, and confirm the previous conclusion. As depicted on Fig. 4, the points much more scattered on the beginner Paddler 3's plot than the case of the professional athletes. Furthermore, the stroke impulse values of Paddler 2's paddling could be divided into two different groups (representing the pulls of the two hands), so the plot indicates the asymmetric style of the athlete. Accordingly, the Poincare plots of stroke time and stroke impulse show obviously the uniformity, distribution and symmetry of the calculated parameters therefore it seems to be an easily usable indicator of the measured performance.

The autocorrelation of the above mentioned parameters fluctuation have been calculated and it shows that the value of the actual stroke has no significant effect on the value of the next stroke, so this fluctuation shows uncorrelated noise, independently the age or technical skills of athletes.

\section{SPECTRAL ANALYSIS OF RAW MOVEMENT SIGNALS}

The relationship between the parameters' fluctuation and the technical skills could be understood, if we consider the fact that steady paddling is required to avoid the suboptimal motion of the kayak. Thus, we have examined the possibility of determining indicators of quality of paddling from the raw movement signals without preprocessing.

The advantage of this method that is not needed to calculate the above mentioned factors with complex detecting algorithms, which could be complicated and inaccurate at a sprint race or a paddling with technical faults.

In Fig. 5 we can see the power density spectrum of the xaxis acceleration and roll axis angular velocity (rotating around the $\mathrm{x}$-axis) measured for the three athletes' typical paddling. The spectrums has been calculated with Hanning window, furthermore it has been smoothed with a 10 samples long moving average and normalized to the dominant frequencies for better visualization. This dominant frequency is the first harmonic (belongs for one stroke cycle) in the case of x-axis acceleration and pitch axis angular velocity (kayaks "nodding"), and it is the fundamental frequency (belongs to the sum of a right and a left hands stroke time) in the case of roll axis and yaw axis angular velocity. The ratio of the magnitude at the first harmonic and the magnitude at the fundamental frequency in the spectrum of $x$-axis acceleration describes the symmetry of the paddling, and the presence of other harmonic
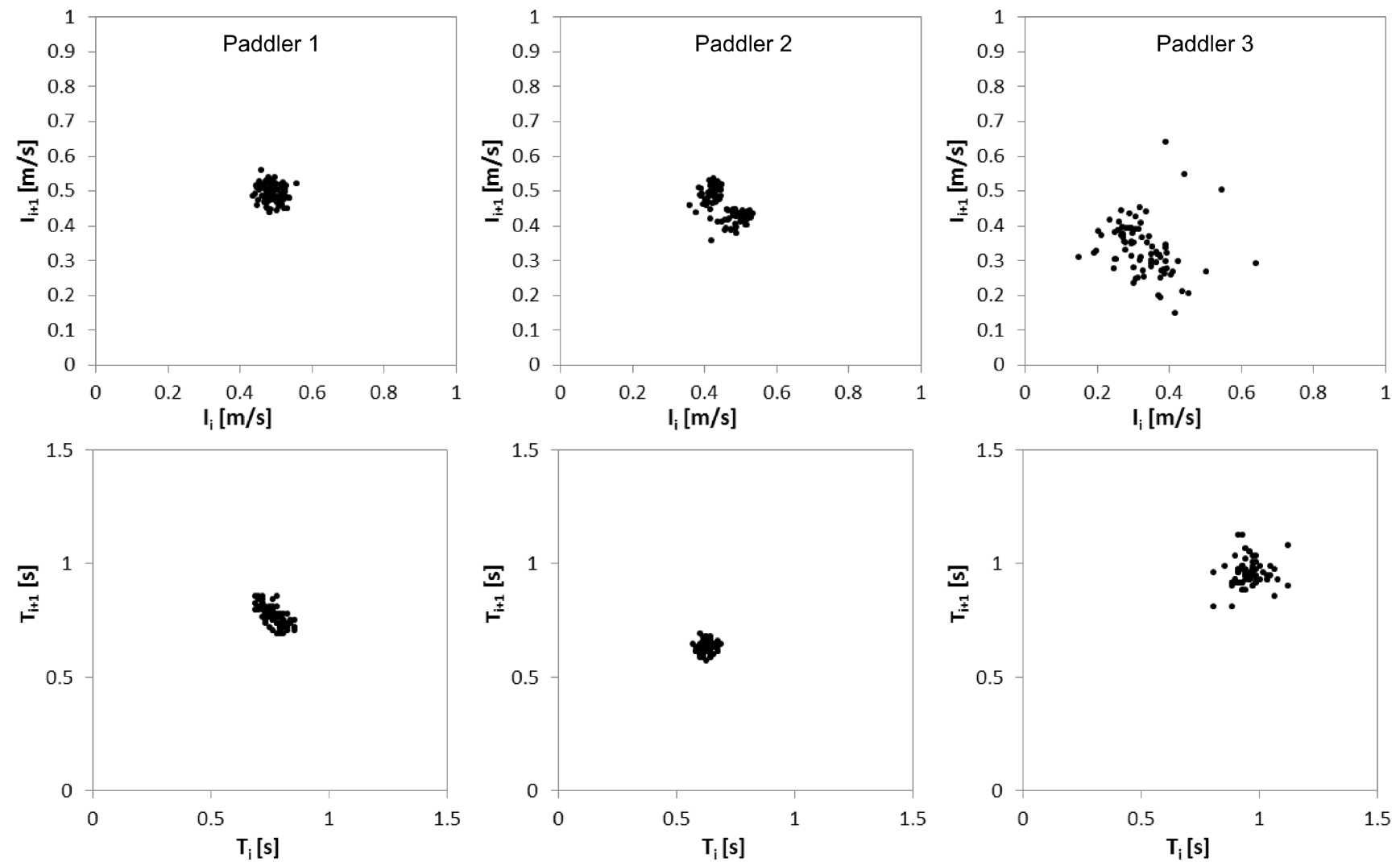

Fig. 4. Poincaré plots of stroke impulse (I, upper row) and stroke time (T, lower row) calculated for three athletes' typical, 80 seconds long paddling without transient phases. 

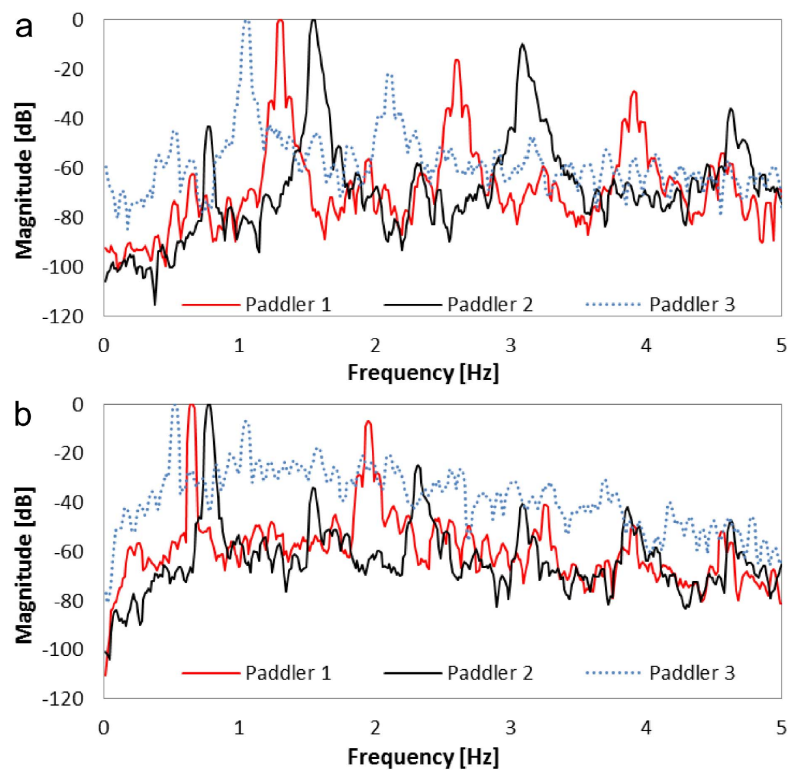

Fig. 5. Power density spectrum of $\mathrm{x}$-axis acceleration (a) and roll axis angular velocity (b) measured at three athletes' typical, 80 seconds

long paddling without transient phases. The spectrums has been calculated using Hanning window, smoothed with a 10 samples long moving average and normalized for the dominant frequencies.

components corresponds to technical faults [6].

The deterministic part of the signal is represented by the harmonic peaks of the normalized power density spectrum and the rest of the spectrum is considered as noise. Fig. 5 shows that the noise level in measured motion signals of Paddler 3's paddling is much greater than the case of professional paddlers. It indicates that the signal to noise ratio (SNR) of raw movement signals indicates the quality of paddling technique, too.

As we can see on Fig. 6, the SNR of roll axis angular velocity increases significantly in the function of the athletes' age. The SNR of other two angular velocities shows this trend too, so it proves our explanation of the relationship between technical skills and parameters' fluctuation, and it provides an indicator of quality of paddling techniques, which could be calculate without using detecting algorithms.

\section{CONCLUSIONS}

Our aim was to find easily usable indicators of paddlers'

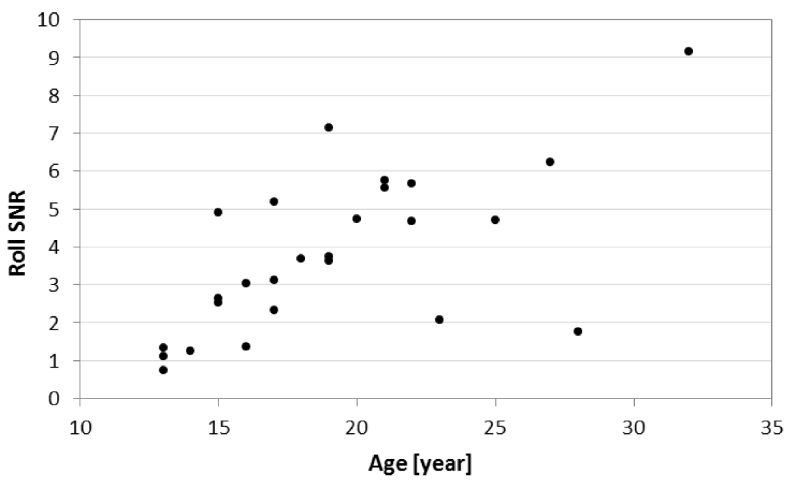

Fig. 6. Signal to noise ratio (SNR) of roll axis angular velocity as a function of paddlers age. technical skills using methods of fluctuation analysis. The analyzed paddlings have been measured by a universal measurement system especially developed for this task [6]. The detailed analysis has been made for 26 athletes of different ages and technical skills.

Using fluctuation analysis of the forward acceleration signal's regular parameters we have found relationship between the standard deviation of stroke time and stroke impulse and the quality of paddling technique. The Poincaré plots of these factors proved to be a spectacular visualization of the steadiness and symmetry of paddlings.

Furthermore, we have shown that the fluctuation of parameters are related to the kayaks suboptimal movements. We have introduced a signal-to-noise ratio analysis calculated from the acceleration and angular velocity signals' power density spectrum that describes the quality of paddling technique as well, additionally this method avoids using complex and less reliable detecting algorithms.

\section{ACKNOWLEDGMENT}

The authors thank P. Kocsis, Prof. G. Szabó, I. Gyémánt, A. Csamangó, A. Dervarics, K. Petrovics and athletes, trainers for their help and valuable discussions.

The publication/presentation is supported by the European Union and co-funded by the European Social Fund. Project title: "Telemedicine-focused research activities on the field of Mathematics, Informatics and Medical sciences" Project number: TÁMOP-4.2.2.A-11/1/KONV-2012-0073.

\section{REFERENCES}

[1] D. A. Aitken, and R. J. Neal, "An on-water analysis system for quantifying stroke force characteristics during kayak events," Int. J. Sport Biomech., vol. 8, pp. 165-173, 1992.

[2] M. G. Robinson, L. E, Holt, T. W. Pelham, and K. Furneaux, "Accelerometry Measurements of Sprint Kayaks: The Coaches' New Tool,” Int. J. Coaching Sci., vol. 5, no. 3, pp. 45-56, January 2011.

[3] Z. Ma, J. Zhang, Y. Sun, and T. Mei, "Sports Biomechanical Information Acquisition and Evaluation for Kayaking Events," Int. J. Inf. Acquisition, vol. 6, no. 3, pp. 213-223, September 2009.

[4] I. Janssen, and A. Sachlikidis, "Validity and reliability of intra-stroke kayak velocity and acceleration using a GPS-based accelerometer," Sport Biomech., vol. 9, no. 1, pp. 47-56, March 2010.

[5] D. R. Pendergast, D. Bushnell, D. W. Wilson, and P. Cerretelli, "Energetics of kayaking," European Journal of Applied Physiology and Occupational Physiology, vol. 59, no. 5, pp. 342-350, December 1989.

[6] G. Vadai, G. Makan, Z. Gingl, R. Mingesz, J. Mellár, T. Szépe and A. Csamangó, "On-water measurement and analysis system for estimating kayak paddlers' performance," in 36th Int. Conv., Microelectronics, Electronics and Electronic Technology, Opatija, 2013, pp. 144-149.

[7] B. Gomes, N. Viriato, R. Sanders, F. Conceição, J. P. Vilas-Boas, and M. Vaz, "Analysis of the on-water paddling force profile of an elite kayaker," in 29th Int. Symp. Biomechanics in Sports, Porto, 2011, pp. 259-262.

[8] R. H. Sanders, and J. S. Kendal, "A description of Olympic Flatwater Kayak Stroke Technique,” Aust. J. Sci. Med. Sport, vol. 24, no. 1, pp. 25-30, 1992.

[9] J. S. Michael, R. Smith, and K. B. Rooney, "Determinants of kayak paddling performance," Sports Biomech., vol. 8, no. 2, pp. 167-179, June 2009. 